\title{
Phosphorrückgewinnung aus Klärschlammaschen mit dem RecoPhos-Prozess
}

\author{
Stefan Windisch · Christoph Ponak · Valentin Mally · Harald Raupenstrauch
}

Online publiziert: 20. Juli 2020

(C) Der/die Autor(en) 2020

\begin{abstract}
Zusammenfassung Um Klärschlämme auch zukünftig in aktuell angewendeten Mitverbrennungsprozessen (z. B. Müllverbrennungsanlagen, Zementwerke) energetisch und stofflich verwerten $\mathrm{zu}$ können, muss eine Möglichkeit zur Rückgewinnung des enthaltenen Phosphors geschaffen werden. Am Lehrstuhl für Thermoprozesstechnik der Montanuniversität Leoben wurde vor einigen Jahren ein Reaktor gebaut - genannt InduRed -, welcher als Herzstück des RecoPhos-Prozesses zur Reduktion

tanuniversität Leoben, den PhosphorRestgehalt in der Schlacke nahezu zu beseitigen bzw. die Anreicherung von Phosphor in der Metallphase zu reduzieren.

Schlüsselwörter Klärschlamm . Phosphor - Carbo-thermische Reduktion

\section{Phosphorus recovery from sewage sludge ashes with the RecoPhos process} von Phosphatverbindungen in Klärschlammaschen dient. Durch das herausfordernde Reaktionsverhalten des Phosphors in Gegenwart von Eisen werden an einen solchen Prozess viele Anforderungen gestellt. Beim RecoPhosProzess werden aus Klärschlammasche mittels carbo-thermischer Reduktion in einer induktiv erwärmten Grafitschüttung mit anschließender Nachverbrennung und Gaswäsche Phosphorsäure, eine phosphorarme Metallphase und eine mineralische Restfraktion erzeugt. Das ambitionierte Ziel der Weiterentwicklung und Verbesserung der Phosphorrückgewinnung ist es, eine mehr als $75 \%$ ige Rückgewinnungsrate zu erzielen und darüber hinaus eine für die stoffliche Verwertung geeignete mineralische Fraktion zu gewinnen. Dies soll durch die Wahl geeigneter Additive und schnelle Abkühlung der Restschlacke nach der Reduktion (z.B. durch Trockenschlackengranulation) passieren. In durchgeführten Versuchskampagnen konnten bereits einige Erfolge erzielt werden. So gelang es beispielsweise in einem kontinuierlichen Versuch in der RecoPhos-Anlage des Lehrstuhls für Thermoprozesstechnik an der Mon-

DI S. Windisch $(\bowtie)$.

DI Dr. C. Ponak · V. Mally ·

Univ.-Prof. DI Dr. H. Raupenstrauch Lehrstuhl für Thermoprozesstechnik, Montanuniversität Leoben, Franz-Josef-Straße 18, 8700 Leoben, Österreich

stefan.windisch@unileoben.ac.at
Abstract In order to continue utilising sewage sludge in currently applied co-incineration processes (e.g. waste incineration plants, cement works) in the future, a possibility to recover the contained phosphorus must be provided. The so-called InduRed reactor, which was developed at the Chair of Thermal Processing Technology at the Montanuniversitaet Leoben, is the crucial element of the RecoPhos process, aiming at the reduction of phosphorus compounds in sewage sludge ashes. Due to the challenging reaction behaviour of phosphorus in the presence of iron, such a process faces a number of challenges. In the RecoPhos process, a high-quality phosphoric acid, a lowphosphorus metal phase and a residual mineral fraction are produced from sewage sludge ash by means of carbothermal reduction in an inductively heated graphite bed with subsequent post-combustion and gas scrubbing. The ambitious goal of the process is the further development and improvement of the phosphorus recovery so that recovery rates over $75 \%$ can be achieved, and to moreover produce a valuable mineral fraction. The latter should be done by choosing suitable additives and rapidly cooling the residual slag after the reduction (e.g. by dry slag granulation). First experimental trials in the RecoPhos plant at the Chair of Thermal Processing Technology at the Montanuniversität Leoben have already been successful. For example, it was possible to reduce the residual phosphorus content in the slag to virtually zero and to limit the accumulation of phosphorus in the metal phase.

Keywords Sewage sludge . Phosphorous · Carbo-thermal reduction

\section{Einleitung}

Da die weltweiten Vorkommen von Phosphor begrenzt sind und Phosphor ein wichtiger Nährstoff und essenzieller Rohstoff für die Düngemittelindustrie ist, gilt dem Phosphorrecycling große Aufmerksamkeit. Aufgrund dieser Bedeutung für die Düngemittel- und Nahrungsmittelindustrie sowie der starken Abhängigkeit von Importen wird Phosphor seit Mai 2014 auch in der Liste kritischer Rohstoffe der EU angeführt. Seit 2017 findet man dort auch Rohphosphat, die wichtigste Primärressource zur Phosphorherstellung (Europäische Kommission 2017). Aufgrund der teils hohen Gehalte und Frachten an Phosphor kommt Klärschlamm dabei eine große Bedeutung zu (Schönberg et al. 2018). Um Stoffkreisläufe innerhalb der EU zu schließen und damit die Importabhängigkeit von Phosphaterzen zu verringern, werden in einigen Ländern bereits Verordnungen vorbereitet bzw. verabschiedet. So ist beispielsweise in Deutschland seit Oktober 2017 die Verordnung zur Neuordnung der Klärschlammverwertung in Kraft, welche schrittweise eine verpflichtende Phosphorrückgewinnung einführt (Bundesministerium für Umwelt, Naturschutz, Bau und Reaktorsicherheit 2017). In Österreich ist zwar noch keine Verordnung in Kraft, jedoch wird im Bundesabfallwirtschaftsplan 2017 bereits eine solche angekündigt. Als aussichtsreichste Verfahrensroute für die Entsorgung von Klärschlamm wird dabei eine Monoverbrennung mit anschließender Phosphorrückgewinnung aus der Asche angesehen (Bundesministerium für Nachhaltigkeit und Tourismus 


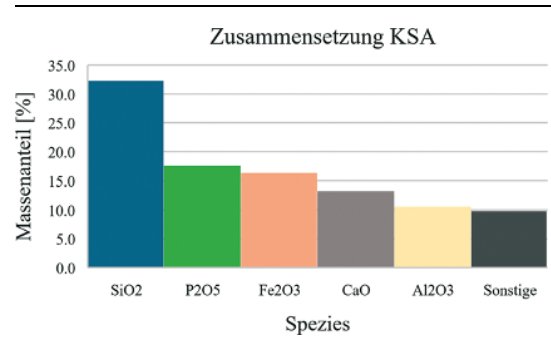

Abb. 1 Typische kommunale Klärschlammaschenzusammensetzung

2017). Da es noch keine großtechnisch einsetzbaren und wirtschaftlichen Verfahren zur Phosphorrückgewinnung gibt, werden die Monoverbrennungskapazitäten jedenfalls stark ausgebaut.

Besonders für die momentan existierende Mitverbrennerindustrie (Müllverbrennung, Zementwerke, Kraftwerke), welche Klärschlamm teilweise als Sekundärbrennstoff bei der Klinkerherstellung oder zur energetischen Verwertung einsetzt, führen diese neuen gesetzlichen Vorgaben zu Herausforderungen. Der aktuelle Entsorgungsweg ist unter den Gesichtspunkten der verpflichtenden Rückgewinnung dann nicht mehr zulässig.

Die Nutzung der im Klärschlamm gebundenen Energie ist in sehr energieintensiven Branchen, wie zum Beispiel der Zementindustrie, im Sinne der Umwelt- und Ressourcenschonung enorm wichtig. Um diese Energie auch in $\mathrm{Zu}$ kunft nutzen zu können und damit Primärrohstoffe einzusparen, gilt es daher, die Phosphorrückgewinnung aus Klärschlamm mit hohen Energienutzungsund Phosphorrückgewinnungsraten zu realisieren.

Dieser Beitrag stellt den sogenannten RecoPhos-Prozess vor, ein Verfahren zur thermischen Rückgewinnung von Phosphor aus Klärschlammasche. Der Fokus des Beitrags liegt dabei auf zwei kürzlich beendeten Versuchskampagnen, welche im Technikum des Lehrstuhls für Thermoprozesstechnik an der Montanuniversität Leoben durchgeführt wurden.

\subsection{Möglicher Verwertungsweg in Zusammenhang mit aktuellen Mitverbrennern}

Klärschlammasche (KSA) aus der Monoverbrennung enthält große Mengen an Phosphor, welcher unter anderem als Kalzium- oder Eisenphosphat (und in Form von Mischphosphaten) vorliegt. Die Asche selbst besteht zum größten Teil aus den oxidischen Komponenten $\mathrm{SiO}_{2}, \mathrm{CaO}, \mathrm{Fe}_{2} \mathrm{O}_{3}$ und $\mathrm{Al}_{2} \mathrm{O}_{3}$, wobei die hohen Eisenoxid- und Aluminiumoxidgehalte durch den Einsatz eisen- und aluminiumhaltiger Fällungssalze in der Abwasserbehandlungsanlage und die anschließende Monoverbrennung zustande kommen. Eine typische KSA-Zusammensetzung aus einer Monoverbrennung ist in Abb. 1 dargestellt.

Abgesehen von der Monoverbrennung bildet auch die Mitverbrennung von Klärschlamm eine Möglichkeit der thermischen Verwertung. So wird Klärschlamm z.B. in der Zementindustrie als Sekundärbrennstoff eingesetzt. Dabei wird Klärschlamm mechanisch entwässert oder im getrockneten $\mathrm{Zu}$ stand im Drehrohrofen mitverbrannt, wodurch energetische Vorteile erzielt werden können. Da die Asche dabei im Klinker eingebaut wird ergibt sich gleichzeitig auch eine vollständige stoffliche Verwertung des Klärschlamms. Dadurch können Rohstoffe und Korrekturstoffe der Zementindustrie eingespart und - im Gegensatz zur Monoverbrennung - die Deponierung der ansonsten anfallenden Aschen vermieden werden (Berger et al. 2010).

Diese Entsorgungsroute erfüllt damit zwar die Grundsätze des Abfallwirtschaftsgesetzes, verhindert auf der anderen Seite aber - durch den Einbau der Asche in die Klinkermatrix - eine Rückgewinnung des enthaltenen Phosphors. Die Verordnung zur Neuordnung der Klärschlammverwertung verlangt aber nach der Rückgewinnung, wodurch die Mitverbrennung im Zementwerk in Zukunft nicht mehr zulässig ist (Bundesministerium für Umwelt, Naturschutz, Bau und Reaktorsicherheit 2017).

Für die Rückgewinnung des Phosphors aus Klärschlamm in einer Müllverbrennungsanlage, einem Kraftwerk bzw. in einem Zementwerk bietet sich aufgrund der vielen Synergiepotenziale besonders ein thermisches Verfahren an. In Abb. 2 ist eine Möglichkeit des Behandlungswegs als Fließbild dargestellt. Der angelieferte, bereits zumindest mechanisch entwässerte Klärschlamm wird einer thermischen Trocknung zugeführt. Die dafür nötige Wärme kann durch Abwärme aus einem Verbrennungsprozess bereitgestellt werden. Anschließend wird der getrocknete Klärschlamm in einer Monoverbrennung bzw. Pyrolyse verascht bzw. pyrolysiert. Es sei erwähnt, dass bisherige Ergebnisse für eine Vera- schung sprechen, wobei auf die dieser Entscheidung zugrunde liegenden Versuche und Auswertungen nicht eingegangen wird und im weiteren Verlauf dieses Beitrags Asche als Ausgangsstoff für die weiteren Schritte betrachtet wird. Die freigesetzte Energie des Veraschungsschritts kann in elektrische Energie umgewandelt und so weiter genutzt werden. Die Klärschlammasche (KSA), welche in diesem Schritt entsteht, wird anschließend der sogenannten RecoPhos-Anlage zugeführt. Das Herzstück dieser Anlage ist der induktiv beheizte Schüttschichtreaktor InduRed. Eine detailliertere Betrachtung der Anlage und des Reaktors folgt etwas später in diesem Beitrag. Wichtig für das Verständnis des Fließschemas ist vorerst, dass die RecoPhos-Anlage drei große Produktströme aufweist: eine Gasphase, welche den Phosphor der KSA enthält und über einen Gasabzug aus dem Reaktor entfernt wird, eine flüssige mineralische Fraktion sowie eine flüssige metallische Fraktion, welche jeweils am unteren Ende des Reaktors gewonnen werden. Die beiden flüssigen Phasen sollen aufgrund ihres Dichteunterschieds getrennt und separat weiterbehandelt werden. Das rückgewonnene Metall besteht dabei zum Großteil aus Eisen und kann potenziell als Legierungsmittel im Bereich der Stahlherstellung bzw. Gießerei recycelt werden. Die metall- und phosphorarme Schlacke soll über die Vermahlung in die Zementherstellung eingebracht und damit stofflich verwertet werden. Es wird auch versucht, durch den Einsatz geeigneter Additive einen Zementzumahlstoff zu erzeugen, welcher in seinen Eigenschaften ähnlich einem zurzeit verwendeten Hüttensand ist. Diese Verfahrenskombination bietet also die Möglichkeit einer reststofffreien Phosphorrückgewinnung aus Klärschlamm in bestehender Mitverbrennerinfrastruktur.

\subsection{Der RecoPhos-Prozess}

Allgemein zielt die thermische Behandlung von KSA darauf ab, die enthaltenen Phosphate $\mathrm{zu}$ reduzieren und Phosphor über die Gasphase zurückzugewinnen. Das Problem an dieser Herangehensweise liegt in der gleichzeitigen Anwesenheit von Eisen und Phosphor in der KSA. Aufgrund der hohen Affinität von Phosphor zu Eisen und der damit einhergehenden Bildung von Eisen-Phosphor-Verbindungen, 


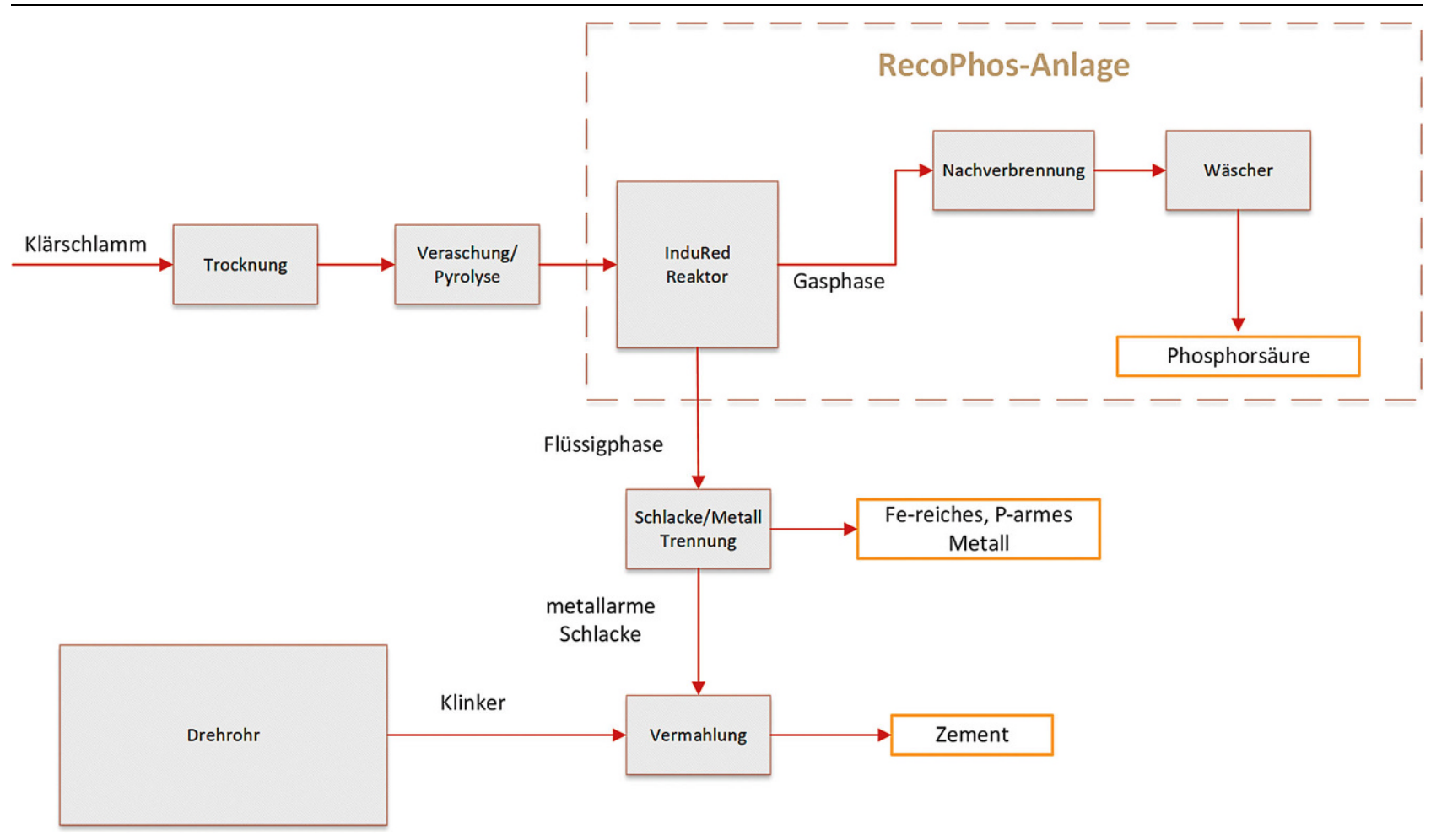

Abb. 2 Möglicher Prozessfluss der Klärschlammbehandlung mit Einsatz des InduRed-Reaktors in bestehender Mitverbrennerinfrastruktur am Beispiel eines Zementwerks

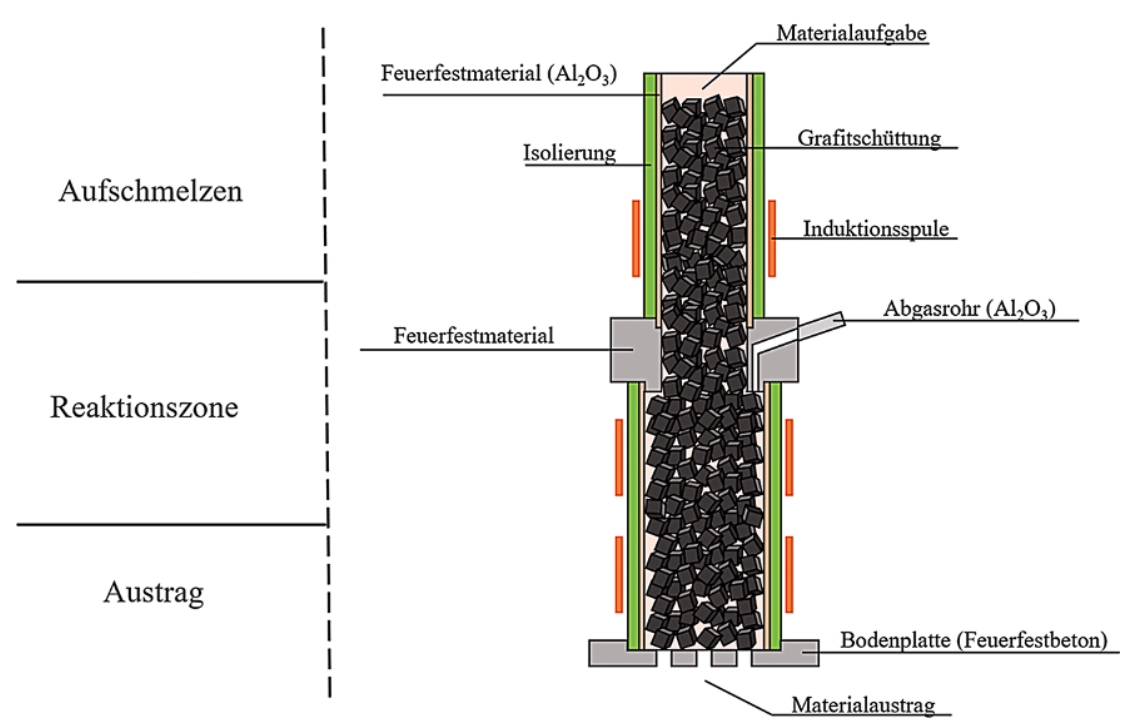

Abb. 3 Schematische Darstellung des InduRed-Reaktors

wird die Rückgewinnungsquote von Phosphor stark eingeschränkt und die Qualität der entstehenden Metallphase verschlechtert. Bevor es zur Bildung solcher Phosphide kommt, liegt Phosphor gelöst in der flüssigen Metallphase vor. Daher muss die Desorption von Phosphor aus der Eisenbasislegierung begünstigt werden. Dies geschieht durch die Einstellung eines Unterdrucks und durch hohe Temperaturen. Letztere begünstigen auch die Unterdrückung der Phosphidbildung (Nakase et al. 2017). Das herausfordernde Reaktionsverhalten von Phosphor führt in bestehenden bzw. vorgestellten thermischen Prozessen dazu, dass geforderte Rückgewinnungsraten nicht eingehalten werden können und schränkt darüber hinaus - durch den hohen Phosphorgehalt auch die Verwertbarkeit der übrigbleibenden Metallphase ein.

Ein Reaktorkonzept, welches diese Einschränkungen potenziell zu umgehen vermag, ist der sogenannte InduRed-Reaktor (Abb. 3). In einem solchen Reaktor werden die enthaltenen Phosphate in einem induktiv beheizten Schüttschicht-Reaktor mittels carbothermischer Reduktion zu elementarem Phosphor reduziert. Der Reaktor besteht aus mehreren, übereinander gestapelten $\mathrm{Al}_{2} \mathrm{O}_{3}$-Keramikringen und einem Mittelteil aus Feuerfestbeton, über welchen ein Gasabzug realisiert ist. Die Keramikringe sind von drei Induktionsspulen umgeben. Innerhalb der Keramikringe befindet sich eine Schüttung aus Grafitbruchstücken. Im Betrieb wird von den Induktionsspulen ein hochfrequentes Wechselfeld hervorgerufen, welches Wirbelströme in die Grafitbruchstücke induziert. Infolge ohmscher Verluste werden die Grafitbruchstücke auf Temperaturen von $1650^{\circ} \mathrm{C}$ erwärmt. Den Reaktor zeichnen dabei zwei wesentliche Eigenschaften aus: Zum einen kann im Reaktor eine sehr sauerstoffarme Atmosphäre mit einem sehr hohen $\mathrm{CO} / \mathrm{CO}_{2}$-Ver- 


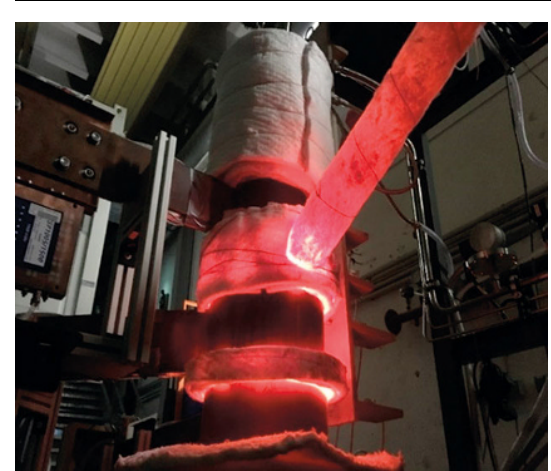

Abb. 4 RecoPhos-Anlage im Betrieb

hältnis aufrechterhalten werden und zum anderen bietet die Schüttung aus Grafitbruchstücken eine große Reaktionsoberfläche.

Das Einsatzgemisch - Klärschlammasche und Additive, wie z.B. CaO und Kohlenstoff - wird kontinuierlich über die Materialaufgabe in den Reaktor chargiert. Das Einsatzgemisch schmilzt dann im oberen Bereich der Schüttung und bewegt sich als dünner Schmelzfilm an den Grafitbruchstücken nach unten. Im mittleren Bereich des Reaktors wird durch die zweite Spule genug Leistung zur Verfügung gestellt, um die Phosphatverbindungen zu reduzieren. Diese Reduktionsreaktionen laufen an der vorhin erwähnten großen Oberfläche der Schüttung ab. Dabei ist anzumerken, dass die Schüttung aus Grafit nur die Oberfläche bietet und dem Prozesskoks als Reduktionsmittel mit dem Aufgabegemisch zugeführt

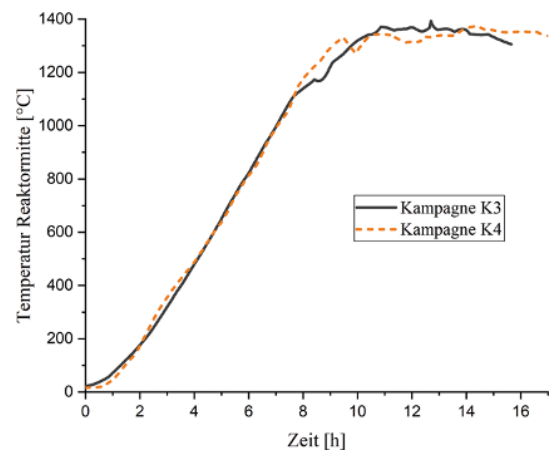

Abb. 5 Aufheizkurven von Versuchskampagnen in der RecoPhos-Pilotanlage (K3 und K4) im Vergleich

wird. Ein weiterer Vorteil der Schüttung ist, dass sich $\mathrm{P}_{2}$, welches nach der Reduktion gasförmig vorliegt, nur durch einen dünnen, sich an den Würfeln entlang bewegenden Schmelzfilm hindurchbewegen muss, bevor es vom Gasabzug erfasst wird. Der Kontakt zwischen Phosphor und Eisen kann dadurch minimiert, die erwähnte Desorption begünstigt und die EisenphosphidBildung $\mathrm{zu}$ einem großen Teil unterdrückt werden. Das Gas, welches durch den Gasabzug abgezogen wird, besteht hauptsächlich aus $\mathrm{CO}$ und $\mathrm{P}_{2}$ und wird in einer Nachbrennkammer unter Luftzufuhr abgebrannt. Das dabei entstehende Phosphorpentoxid soll dann in einem Wäscher ausgewaschen und zu Phosphorsäure hydrolysiert werden. Die Schlacken- und Metallphasen durchqueren den Reaktor weiter nach unten, bis sie ihn über einen argongespülten Materialaustrag verlassen. Die dritte Spule liefert genug Energie, um die Temperatur der flüssigen Phasen hoch zu halten, damit eine niedrige Viskosität aufrecht zu erhalten und einen flüssigen Austrag zu ermöglichen. Die flüssigen Phasen werden danach in einem Behälter gesammelt und können getrennt und weiter untersucht werden.

\section{Versuche}

Die eben beschriebene RecoPhos-Anlage wird am Lehrstuhl für Thermoprozesstechnik an der Montanuniversität betrieben, untersucht und ständig weiterentwickelt. Sie ist für einen kontinuierlichen Materialeintrag von $5 \mathrm{~kg} / \mathrm{h}$ ausgelegt und wird abgesehen von der Behandlung von Klärschlammasche auch für Stahlwerksschlacken und weitere industrielle oder kommunale Reststoffe eingesetzt. In vergangenen Versuchskampagnen wurden einige Verbesserungen - z. B. ein überarbeiteter Gasabzug, um den EisenPhosphor-Kontakt weiter zu verringern, oder ein neues Austragskonzept in der Anlage umgesetzt und getestet. Die nachfolgend beschriebenen und zuletzt durchgeführten Kampagnen K3 (Abb. 4) und K4 hatten das Ziel, den Einfluss der durchgeführten Anlagenverbesserungen auf die P-Rückgewinnungsquote $\mathrm{zu}$ untersuchen und die Phosphorbilanz in der Anlage durch eine Gasmessung in der K4 zu schließen. Dabei wurde ein Teilgasstrom nach der Nachbrennkammer abgesaugt und durch unterschiedliche Waschmedien (sauer/basisch) gereinigt.

Tab. 1 Zusammensetzung der Eintragsgemische für zwei Versuchskampagnen (K3/K4) in der RecoPhos-Pilotanlage und dosierte Mengen

\begin{tabular}{|l|l|l|l|}
\hline Spezies & Massengehalt im Feed [\%] & Massen K3 [g] & Massen K4 [g] \\
\hline $\mathrm{CaO}$ & 25,04 & 2724,22 & 3004,65 \\
\hline $\mathrm{SiO}_{2}$ & 25,04 & 2724,22 & 3004,65 \\
\hline $\mathrm{P}_{2} \mathrm{O}_{5}$ & 13,64 & 1484,40 & 1637,21 \\
\hline $\mathrm{Fe}_{2} \mathrm{O}_{3}$ & 12,71 & 1383,19 & 1525,58 \\
\hline $\mathrm{Al}_{2} \mathrm{O}_{3}$ & 8,22 & 894,02 & 986,05 \\
\hline $\mathrm{C}$ & 7,75 & 843,41 & 930,23 \\
\hline $\mathrm{MgO}$ & 2,61 & 284,23 & 313,49 \\
\hline $\mathrm{SO}_{3}$ & 1,50 & 162,78 & 179,53 \\
\hline $\mathrm{K}_{2} \mathrm{O}$ & 1,33 & 144,22 & 159,07 \\
\hline $\mathrm{TiO}_{2}$ & 0,70 & 76,16 & 84,00 \\
\hline $\mathrm{Na}_{2} \mathrm{O}$ & 0,40 & 44,03 & 48,56 \\
\hline $\mathrm{MnO}_{2}$ & 0,11 & 12,15 & 13,40 \\
\hline $\mathrm{SrO}_{2}$ & 0,09 & 9,53 & 10,51 \\
\hline $\mathrm{BaO}$ & 0,08 & 8,60 & 9,49 \\
\hline $\mathrm{Sonstige}$ & 0,78 & 84,85 & 93,58 \\
\hline Gesamt & - & 10,880 & 12.000 \\
\hline
\end{tabular}




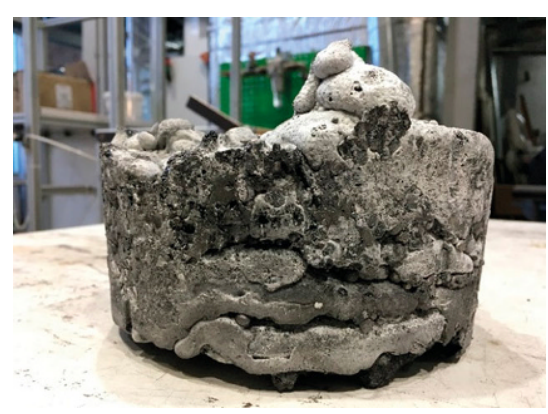

Abb. 6 Schlacke aus der carbo-thermischen Reduktion von Klärschlammasche im InduRed-Reaktor

Um die Ergebnisse von Kampagne K3 und K4 vergleichen zu können, wurde versucht, sie unter möglichst gleichen Bedingungen durchzuführen. Die Zusammensetzung des Einsatzgemisches und die jeweils dosierten Mengen sind Tab. 1 zu entnehmen.

Bei den durchgeführten Kampagnen wurde die Anlage in einem 8-stündigen Aufheizvorgang auf eine ReaktorInnentemperatur von $1650^{\circ} \mathrm{C}$ erhitzt. Die Temperaturmessung erfolgte dabei über Typ-S-Thermoelemente an der Reaktoraußenwand. In Abb. 5 ist zu erkennen, dass der Aufheizvorgang nahezu ident abgelaufen ist. Dem Aufheizvorgang folgte dann eine etwa 5- bzw. 7-stündige Dosierphase bei annähernd konstanter Temperatur, in welcher jeweils über $10 \mathrm{~kg}$ des Einsatzgemisches dosiert wurden.

\subsection{Ergebnisse}

Die in der Kampagne K3 gewonnenen Schlacken- und Metallphasen sind in Abb. 6 dargestellt. Insgesamt konnte erstarrtes Material mit einer Masse von $9967 \mathrm{~g}$ gesammelt werden, wovon $8697 \mathrm{~g}$ auf Schlacke und $1296 \mathrm{~g}$ auf die Metallphase entfielen. In der Kampagne K4 wurden insgesamt $9134 \mathrm{~g}$ Material mit einer ähnlichen Verteilung - 7842 g Schlacke und 1292g Metall - produziert. Hierbei ist anzumerken, dass aufgrund der schwierigen Probenahme die Metallmengen jeweils aus den aus Analysen bestimmten Reduktionsgraden berechnet wurden.

Bei der Probenahme wurden mehrere Zonen der Probe definiert, diese Zonen einzeln beprobt und daraus eine repräsentative Mischprobe hergestellt. Dies wurde für beide Produktströme, also jeweils für Schlacken- und $\mathrm{Me}$ -

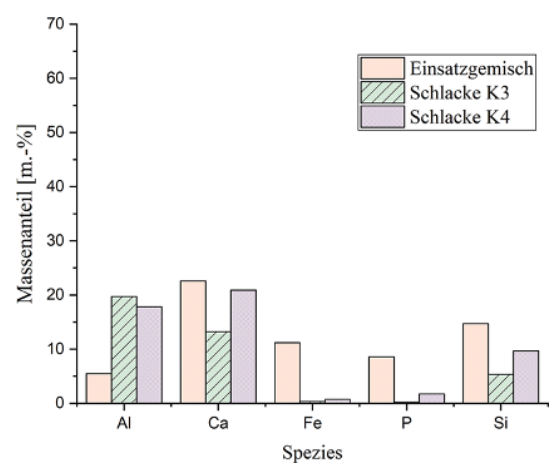

Abb. 7 Zusammensetzung der Schlackenphase aus der carbo-thermischen Reduktion von Klärschlammasche im InduRed-Reaktor

tallphase, durchgeführt und mittels ICP-MS analysiert. Die Ergebnisse der Analyse der durchschnittlichen Schlackenzusammensetzung sind in Abb. 7 dargestellt.

Aus den Ergebnissen der Schlackenanalyse lassen sich mehrere Schlüsse ziehen. Der durch einen chemischen Angriff auf das Feuerfestmaterial ausgelöste Aluminiumoxideintrag während des Versuchs ist sehr hoch und führt zu deutlich erhöhten Aluminium-Werten. Diese Anreicherung ist grundsätzlich dem Anlagendesign in dieser ScaleStufe geschuldet. Konzepte für eine Reduktion des Aluminiumeintrags wurden bereits untersucht und im nächsten Anlagenscale implementiert, können in dieser Anlagengröße allerdings schwer umgesetzt werden. Bemerkenswert sind die Werte für den Eisen- bzw. Phosphorgehalt in der Schlacke nach der Reduktion. In der Kampagne K3 konnte der Eisengehalt in der Schlacke auf unter 1,5 m.-\%, der Phosphorgehalt sogar auf unter $0,3 \mathrm{~m}$.-\% gesenkt werden. Die Kampagne K4 weist besonders beim Phosphorgehalt (1,7 m.-\%) einen deutlich höheren Wert auf. Dies wurde auf die abschnittsweise zu niedrige Reduktionstemperatur zurückgeführt, da versucht wurde, das Feuerfestmaterial durch Vermeidung von Temperaturspitzen zu schonen. Tatsächlich ist der AlEintrag geringer als in K3, jedoch muss gewährleistet sein, dass die Reduktion vollständig abläuft.

Noch größer fällt der Unterschied zwischen K3 und K4 bei der Analyse der Metallphase (Abb. 8) auf.

In der Abbildung ist zu erkennen, dass der Phosphorgehalt der Metallphase in der Kampagne K4 beinahe doppelt so hoch wie in der Kampagne K3 ist. Dies deutet darauf hin, dass die

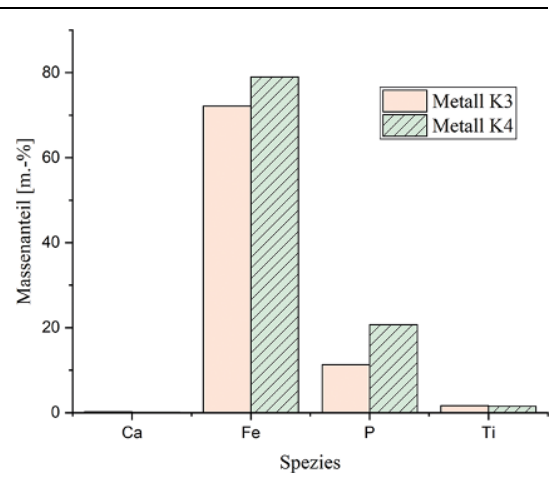

Abb. 8 Zusammensetzung der Metallphase aus der carbo-thermischen Reduktion von Klärschlammasche im InduRedReaktor

Eisenphosphidbildung in K4 weniger gehemmt wurde als in der vorherigen Kampagne. Dies stimmt gut mit thermodynamischen Betrachtungen und der niedrigeren Reaktortemperatur als identifizierte Ursache überein. Um einen Einfluss von Schlackeneinschlüssen auf die Analysenergebnisse auszuschließen, wurde der Kalziumgehalt in der Metallphase mitanalysiert. Die niedrigen Kalziumgehalte zeigen, dass in der Metallprobe keine Schlackenbestandteile mitgemessen wurden.

\subsection{Diskussion}

Da eine vollständige Massenbilanz aufgrund der feinen Verteilung der Metalllegierung in der erstarrten Schlacke und der fehlenden Gaserfassung nicht möglich ist, wurden Metall- und Gasmenge iterativ mithilfe der Analysen berechnet.

Für den Verbleib des restlichen Phosphors in K3 - nur $25 \mathrm{~m}$.-\% der eingebrachten Menge wurden in der Schlacke und dem Metall detektiert - ergibt sich aus thermodynamischer Sicht keine andere Möglichkeit als die Gasphase. Dies geht auch aus vorhandener Literatur hervor (Matinde et al. 2008). Die hohen Temperaturen und der niedrige Sauerstoffpartialdruck führen zu einer ausreichend reduzierenden Atmosphäre, in welcher $\mathrm{P}_{2} \mathrm{O}_{5}$ jedenfalls zu gasförmigem, elementarem Phosphor (Siedepunkt $280^{\circ} \mathrm{C}$ ) umgesetzt wird. Ein weiterer Indikator für die Produktion von $\mathrm{P}_{2}$ und $\mathrm{CO}$ ist die Korrelation zwischen Brennkammertemperatur und Materialeintrag (Abb. 9).

Hierbei ist zu sehen, dass die Brennkammertemperatur ansteigt, sobald Material in den Reaktor chargiert wird. Dies lässt sich durch die Reduktion der 


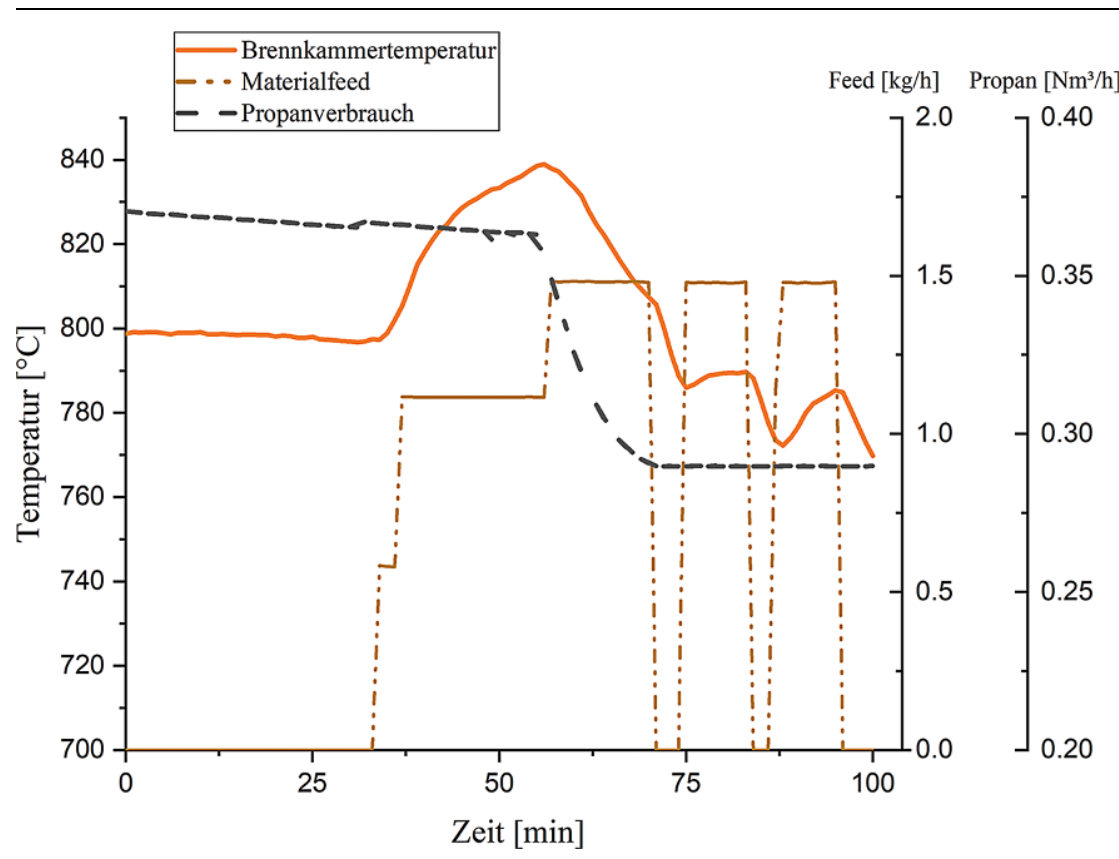

Abb. 9 Brennkammertemperatur während der Gemischdosierung bei der Behandlung von Klärschlammasche in der RecoPhos-Pilotanlage

\section{Gaszusammensetzung nach dem Reaktor [Vol.-\%]}

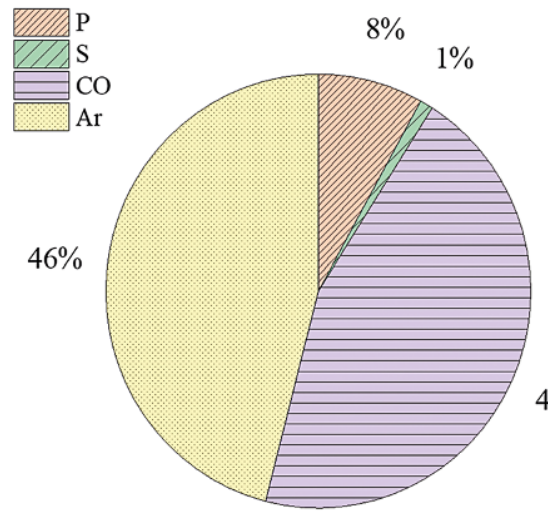

mit Argon

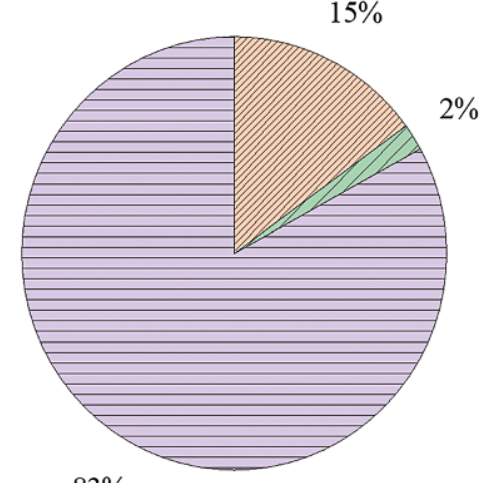

$83 \%$ ohne Argon

Abb. 11 Berechnete Gaszusammensetzung nach dem Reaktor in K3 (carbo-thermische Reduktion von Klärschlammasche im InduRed-Reaktor)

enthaltenen Oxide im Reaktor erklären. Bildet sich infolge indirekter Reduktion (untergeordnete Rolle) $\mathrm{CO}_{2}$ oder dringt Falschluft ins System, so wird das Gas entsprechend dem BoudouardGleichgewicht mit dem ausreichend vorhandenen Kohlenstoff zu CO umgesetzt. Gelangen dann $\mathrm{CO}$ und $\mathrm{P}_{2}$ über den Gasabzug in die Brennkammer, reagieren die Gase exotherm mit dem Sauerstoff der eingebrachten Luft. Dies führt dann zu einer Erhöhung der Brennkammertemperatur, welche die Anlage mit einer Reduzierung der
Propanzufuhr (zum Aufheizen verwendet) kompensiert. Zusätzlich ist bei der wiederholten Messung des $\mathrm{pH}$-Werts und der Orthophosphat-Konzentration im Waschwasser zu erkennen, dass die Entfernung von Phosphor über die Gasphase erfolgt.

Dies lässt den Schluss zu, dass jener Teil des eingebrachten Phosphors, welcher sich nicht in der Schlacke oder Metallphase angereichert hat, in der Gasphase aus dem Reaktor entfernt wurde. Daraus ergibt sich für die Kampagne K3 folgende Phosphorverteilung (Abb. 10).

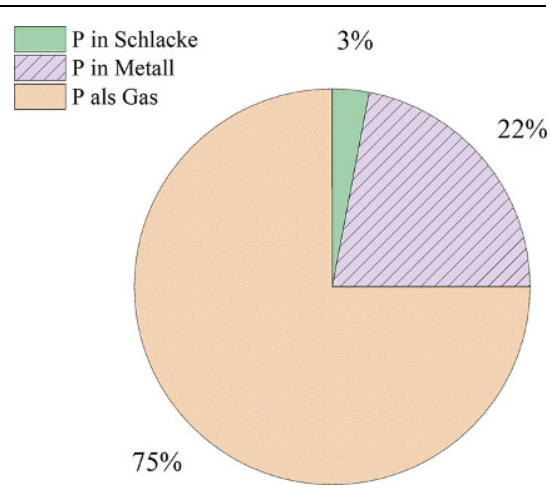

Abb. 10 Berechnete Verteilung des Phosphors auf die Produktströme in K3 (carbo-thermische Reduktion von Klärschlammasche im InduRed-Reaktor)

Einen genaueren Überblick über die Zusammensetzung der Gasphase aus dem Reaktor bietet Abb. 11. Es ist zu erkennen, dass das zur Vermeidung von Re-Oxidationsreaktionen beim Materialaustrag bzw. zur Vermeidung einer sauerstoffreichen Atmosphäre beim Materialeintrag eingebrachte Argon einen großen Teil der Gasphase ausmacht. Berechnet man nur die aus der KSA entstehende Gasphase, so besteht diese zu mehr als 80 Vol.- $\%$ aus CO. Eine energetische Nutzung der Gasphase ist also durchaus denkbar und könnte im Energieverbund eines Verbrennungsprozesses genutzt werden.

Die Ergebnisse der in K4 durchgeführten Gasmessung zeigten einige Unstimmigkeiten. Es wurde weniger Phosphor in den Waschmedien gefunden als zuvor berechnet. Der - vermutlich durch die niedrigere Reaktionstemperatur ausgelöste - schlechtere Reduktionsgrad der KSA führte zu niedrigeren Wiederfindungsraten als zuvor aus der Berechnung für die Gaszusammensetzung ermittelt wurde. Bei genauer Betrachtung der Analysen aus der Gasmessung und Berücksichtigung der höheren Phosphoranreicherung in der Metallphase bei der K4 konnte abschließend der hohe Transferkoeffizient von Phosphor in die Gasphase bestätigt werden. Abb. 12 zeigt ein SankeyDiagramm bezogen auf $100 \%$ Produktströme.

\section{Schlussfolgerung und Ausblick}

Es konnte durch Kampagnen in der RecoPhos-Pilotanlage noch vor jedweder Optimierung hinsichtlich einer großtechnischen Anwendung (Feuerfestmaterial-Optimierung, Positionie- 


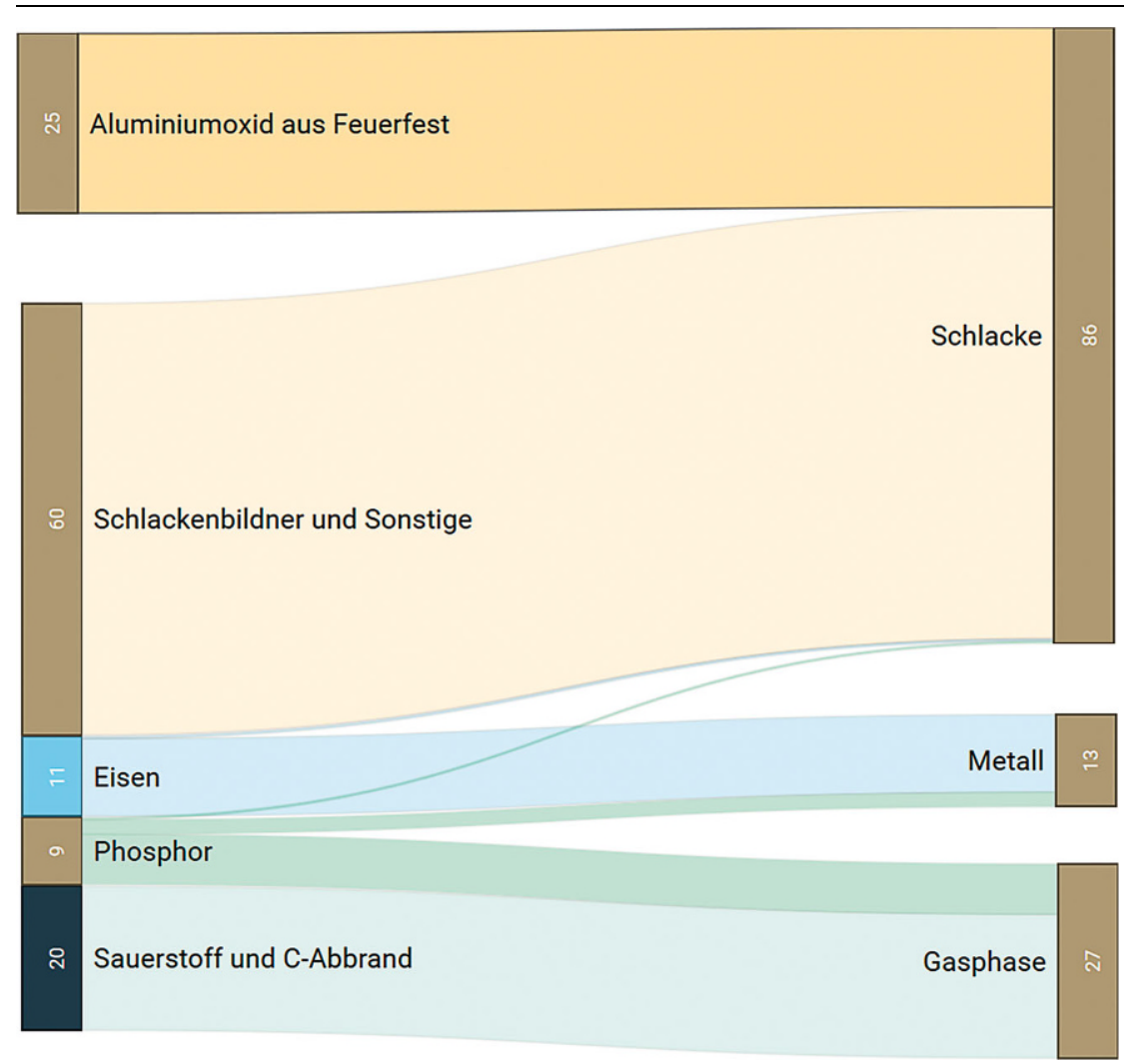

Abb. 12 Aufteilung auf die Produktströme bei der carbo-thermischen Reduktion von Klärschlammasche im InduRed-Reaktor (als Teil des RecoPhos-Prozesses)

rung mehrerer Gasabzüge, Einstellung eines höheren Unterdrucks etc.) gezeigt werden, dass die vollständige Reduktion von Phosphaten aus Klärschlammaschen und der Transfer des elementaren Phosphors in die Gasphase zu einem hohen Prozentsatz möglich sind. Die entstehende Gasphase kann zuerst energetisch verwertet und das enthaltene $\mathrm{P}_{2} \mathrm{O}_{5}$ anschließend in einem Wäscher zu Phosphorsäure umgesetzt werden. Die Schlacke kann in ihrer Zusammensetzung so eingestellt werden, dass bei rascher Erstarrung eine hüttensandähnliche, glasige Struktur entsteht, welche optimal als Zumahlstoff in der
Zementherstellung verwendet werden kann.

Die Versuche, welche an der Montanuniversität Leoben durchgeführt wurden, weisen bereits jetzt eine Rückgewinnungsrate von $75 \%$ des Phosphors aus der KSA auf. Diese Raten konnten durch eine ständige Weiterentwicklung der bestehenden Anlage erreicht und durch die Versuchskampagnen K3 und K4 bestätigt werden. Um neue Konzepte, beispielsweise einen Schutz des Feuerfestmaterials oder eine Trennung von Schlacke und Metall in der Flüssigphase, erproben und untersuchen zu können, wird aktuell an einem Scale-Up der bestehenden Anla- ge gearbeitet. Dabei sollen die Desorption von Phosphor aus der Eisenbasislegierung optimiert, die Umsetzung des elementaren Phosphors zur Säure und der Angriff des Feuerfestmaterials genauer untersucht werden.

Förderung Diese Arbeit wurde durch Mittel des Bundesministeriums für Verkehr, Innovation und Technologie im Rahmen des FFG-geförderten Programms „Produktion der Zukunft“ unter der Fördernummer 864855 ermöglicht. Für diese Förderung sei an dieser Stelle ausdrücklich gedankt.

Funding Open access funding provided by Montanuniversität Leoben.

Open Access Dieser Artikel wird unter der Creative Commons Namensnennung 4.0 International Lizenz veröffentlicht, welche die Nutzung, Vervielfältigung, Bearbeitung, Verbreitung und Wiedergabe in jeglichem Medium und Format erlaubt, sofern Sie den/die ursprünglichen Autor(en) und die Quelle ordnungsgemäß nennen, einen Link zur Creative Commons Lizenz beifügen und angeben, ob Änderungen vorgenommen wurden.

Die in diesem Artikel enthaltenen Bilder und sonstiges Drittmaterial unterliegen ebenfalls der genannten Creative Commons Lizenz, sofern sich aus der Abbildungslegende nichts anderes ergibt. Sofern das betreffende Material nicht unter der genannten Creative Commons Lizenz steht und die betreffende Handlung nicht nach gesetzlichen Vorschriften erlaubt ist, ist für die oben aufgeführten Weiterverwendungen des Materials die Einwilligung des jeweiligen Rechteinhabers einzuholen.

Weitere Details zur Lizenz entnehmen Sie bitte der Lizenzinformation auf http://creativecommons.org/licenses/ by/4.0/deed.de. 


\section{Originalarbeit}

\section{Literatur}

Berger, H., V. Hoenig, Z. Aslan, G. Bachmann, T. Eisenhut, O. Knöpfelmacher et al. (2010): Energieeffizienz der österreichischen Zementindustrie, Vereinigung der Österreichischen Zementindustrie, Wien

Bundesministerium für Nachhaltigkeit und Tourismus (2017): Bundesabfallwirtschaftsplan 2017, Wien

Bundesministerium für Umwelt, Naturschutz, Bau und Reaktorsicherheit (2017): Verordnung zur Neuordnung der Klärschlammverwertung, AbfKlärV. In : Bundesgesetzblatt 2017 Teil 1 Nr. 65 Europäische Kommission (2017): Mitteilung der Kommission an das europäische Parlament, den Rat, den europäischen Wirtschafts- und Sozialausschuss und den Ausschuss der Regionen über die Liste kritischer Rohstoffe für die EU, https://ec.europa.eu/transparency/regdoc/ rep/1/2017/DE/COM-2017-490-F1-DE-MAIN-

PART-1.PDF. Zugegriffen: 21.04.2020

Matinde, E., Y. Sasaki and M. Hino (2008): Phosphorus Gasification from Sewage Sludge during Carbothermic Reduction, ISIJ International, 48, 912-917

Nakase, K., A. Matsui, N. Kikuchi and Y. Miki (2017): Effect of Slag Composition on Phosphorus Separation from Steelmaking Slag by Reduction, ISIJ International, 57, 1197-1204
Schönberg, A., H. Raupenstrauch and C. Ponak (2018): Verfahren und Produkte der Phosphorrückgewinnung, Berliner Abfallwirtschafts- und Energiekonferenz, Berlin, 2018, 679-691

Hinweis des Verlags Der Verlag bleibt in Hinblick auf geografische Zuordnungen und Gebietsbezeichnungen in veröffentlichten Karten und Institutsadressen neutral. 